\title{
Allozyme variation in the marine fanworm Sabella spallanzanii: comparison of native European and introduced Australian populations
}

\author{
Jane Andrew, Robert D. Ward* \\ CSIRO Division of Marine Research, GPO Box 1538, Hobart, Tasmania 7001, Australia
}

\begin{abstract}
Three Australian (Cockburn Sound, Western Australia; Adelaide, South Australia; Port Phillip Bay, Victoria) and four European (Roscoff, Atlantic; Alicante, Carteau, and Marsellle, all from the Mediterranean Seaj populations of Sabella spallanzanil were examined for variation at 23 allozyme loci. Levels of genetıc variation were high, with average heterozygosities per locus ranging from 0.20 to 0.21 for the Australian collections, and from 0.23 to 0.27 for the European collections. The 3 Australian collections were genetically very closely related to one another, with no statistically significant differences at any of the 15 variable locl. This suggests either an introduction to one port, with a subsequent spread to other areas, or separate introductions with subsequent extensive gene flow. The 4 European collections showed limited, but significant, differentiation among themselves at 10 of 18 variable loci. Much of the heterogeneity reflected differences between the Roscoff (Atlantic) collection and the 3 Mediterranean collections. The Roscoff collection was less variable, in terms of both heterozygosity and mean numbers of alleles, than the Mediterranean collections. The Australian collections were more closely related to the Mediterranean collections (mean Nei distance $=0.040$ ) than to the Roscoff collection (mean Nei distance $=0.084$ ). If a Mediterranean origin is assumed, then the introduction to Australia was accompanied by the loss of about $18 \%$ of the original variation. Finally, the high genetic similarity of the Australian to the European collections of $S$. spallanzanii, and the very clear genetic separation of $S$ pavonina (mean Nei distance $=0.996$ ), reaffirms that the Australian collections are truly $S$ spallanzanii and not an undescribed native species.
\end{abstract}

KEY WORDS: Sabella Allozyme - Polymorphism - Genetic differentiation Founder effect Introduction

\section{INTRODUCTION}

Sabella (Spirographis) spallanzanii is a marine fanworm, a member of the family Sabellidae, class Polychaeta. Most publications referring to this species refer to it as Spirographis spallanzanii Viviani (1805), but it appears to have been recognised earlier as Sabella spallanzanii Gmelin 1791. Ewer (1946) synonymised the genera Spirographis and Sabella, and recently Perkins \& Kright-Jones (1991) synonymised Spirographis spallanzanii Viviani with Sabella penicillus Linnaeus, but suggested suppressing penicillus and

- Addressee for correspondence.

E-mail:bob.ward@marine.csiro.au other synonyms to stabilise spallanzanil. That is the nomenclature we will use.

This species is widespread in the Mediterranean, being recorded from the coasts of Spain, France, Italy, Turkey and Morocco. It is also found on eastern Atlantic coasts, e.g. Morocco (Bitar 1987), from Portugal (H. Zibrowius pers. comm.) to northwest France, and in the Azores. There is a record of an isolated, presumably introduced, population in Rio de Janeiro (P Knight-Jones pers. comm. referred to in Carey \& Watson 1992). It is found attached to the substrate in waters from 1 to $30 \mathrm{~m}$ in depth, and is particularly prevalent in harbours, where it can rapidly colonise human-made structures (Koechlin 1977. Clapin \& Evans 1995). 
Sabella spallanzanii was first reported from Australian waters in the Geelong arm of Port Phillip Bay, Victoria (Carey \& Watson 1992). Although it had not been seen in this region prior to the early 1980 s, by 1991 it had become common, the visually dominant organism of the muddy bottom habitat', releasing 'masses of green-coloured eggs' in late summer (Carey \& Watson 1992). Scallop fishers in the Geelong arm first noticed the fanworm in their catches in 1993; fanworms now clog dredges and increase catch-sorting times. The fanworm has also hindered the snapper fishery through interference with longlines.

In September 1994, dense beds of sabellid worms were found in Cockburn Sound (Western Australia). However, aerial photographs from 1983 showed probable fanworm patches, and there were anecdotal reports and a photograph of what now appear to be specimens of Sabella spallanzanii in 1985 (Clapin \& Evans 1995). Subsequently (March 1995), 2 Aỉbany (Western Australia) harbours were surveyed, and $S$. spallanzanii was found in both. Sabellids in the Western Australian Museum include 3 putative specimens of $S$ spallanzanii, collected from Albany in 1965, 1978 and 1979. Thus this fanworm may have been present in this region for at least $30 \mathrm{yr}$ (Clapin \& Evans 1995). In 1995, large numbers of $S$. spallanzanii were found near Adelaide, but South Australian Museum records indicate that the species may have been present since at least the mid-1970s (S. Rainer pers. comm.).

Sabella spallanzanii is now widespread and abundant in many parts of southern Australia. It is presumed to have been introduced either as a fouling organism on ships' hulls or possibly through the release of ballast water containing larvae or young juveniles. Once established in a suitable habitat, it can proliferate rapidly, reaching high densities. Reproduction is sexual, and large numbers of lecithotropic (nonfeeding) larvae are produced (Giangrande \& Petraroli 1994, Rouse \& Fitzhugh 1994). Colonies may compete directly with native species for space and food and, by stripping phytoplankton from the water, may change the ecology of the invaded area. Its impact on fisheries in Port Phillip Bay has been significant, although in Cockburn Sound Clapin \& Evans (1995) found no evidence either that the fanworm was a direct threat to any fishery there or that it had any apparent impact on native species.

The aim of this study was to throw some light on the genetics of the species, in particular to confirm that the Australian populations really are Sabella spallanzaniu, to determine the genetic relationships among Australian populations, and to see how closely related they are to European populations. There have been no previous genetic studies of this species, either in Australia or elsewhere.

\section{METHODS}

Collections of samples. In Australia, collections of Sabella spallanzanii were made from 3 states (Western Australia, South Australia and Victoria). In Europe, Mediterranean collections came from France (Marseille and nearby Carteau) and Spain (Alicante), and an Atlantic collection came from France (Roscoff). Further details are provided in Table 1 and Fig. 1 . One collection $(n=10)$ of Sabella pavonina was made, from Roscoff. This species was to serve as an outgroup in phylogenetic trees, and to provide an indication of the extent of genetic differentiation within the genus Sabella.

After collection, the worms were removed from their tubes, and the anterior $10 \mathrm{~cm}$ wrapped in aluminium foil and frozen. The Mediterranean samples were stored at -20 to $-35^{\circ} \mathrm{C}$ for several weeks and Atlantic samples stored in liquid nitrogen for a few days before being airfreighted in dry-ice to Hobart. The Australian samples were dispatched immediately to Hobart,

Table 1 Sampling sites and dates for collection of Sabella spallanzanii. In addition to 62 S. spallanzanii, 10 Sabella pavonina were also collected at Roscoff in January 1996

\begin{tabular}{|c|c|c|c|c|}
\hline Location & Description & Date & Sample size & Latitude, longitude \\
\hline \multicolumn{5}{|l|}{ Australia } \\
\hline Victoria & Point Wilson Pier, near Geelong, Port Phillip Bay & Sep 1995 & 108 & $38^{\circ} 5^{\prime} \mathrm{S}, 144^{\circ} 32^{\prime} \mathrm{E}$ \\
\hline South Australia & North Haven Boat Harbour, Adelaide & Nov 1995 & 100 & $34^{\circ} 47^{\prime} \mathrm{S}, 138^{\circ} 29^{\prime} \mathrm{E}$ \\
\hline Western Australia & Southern Flats, Cockburn Sound, near Fremantle & Dec 1995 & 100 & $32^{\circ} 5^{\prime} \mathrm{S}, 115^{\circ} 42^{\prime} \mathrm{E}$ \\
\hline \multicolumn{5}{|l|}{ Europe } \\
\hline Roscoff & Car ferry port & Jan 1996 & 62 & $48^{\circ} 43^{\prime} \mathrm{N}, 3^{\circ} 58^{\prime} \mathrm{W}$ \\
\hline Carteau & Golfe de Fos, Rhone River estuary & Oct 1995 & 48 & $43^{\circ} 22^{\prime} \mathrm{N}, 4^{\circ} 52^{\prime} \mathrm{E}$ \\
\hline Marseille & Marseille Harbour & Oct 1995 & 50 & $43^{\circ} 19^{\prime} \mathrm{N}, 5^{\circ} 21^{\prime} \mathrm{E}$ \\
\hline \multirow[t]{2}{*}{ Alicante } & Cabo Roig (Red Cape), Torrevieja (natural site); and & Dec 1.995 & 30 & $37^{\circ} 54^{\prime} \mathrm{N}, 0^{\circ} 43^{\prime} \mathrm{W}$ \\
\hline & Torrevieja Harbour (polluted) & Dec 1995 & 30 & $37^{\circ} 56^{\prime} \mathrm{N}, 0^{\circ} 41^{\prime} \mathrm{W}$ \\
\hline
\end{tabular}


(a)

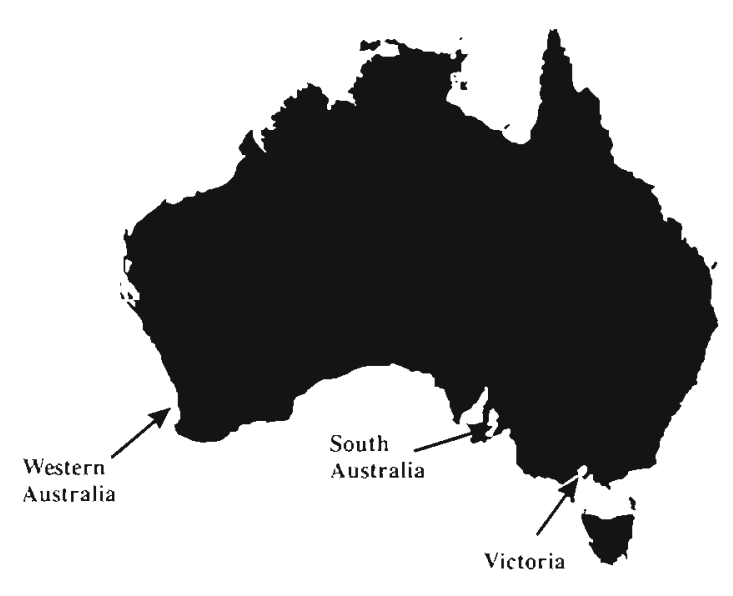

(b)

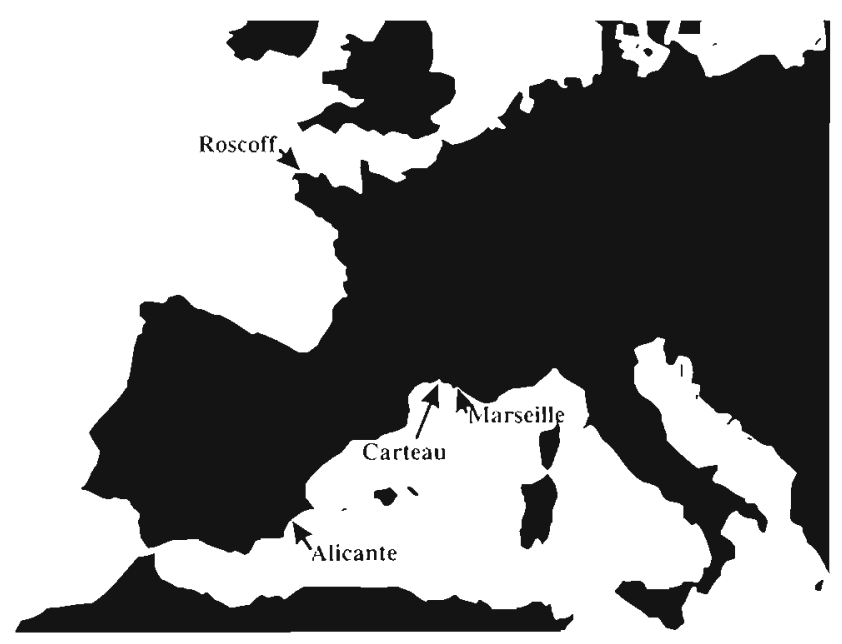

Fig. 1. Sites of collection of marine fanworms in (a) Australia and (b) Europe

either in liquid nitrogen (Victoria) or on dry-ice (South Australia, Western Australia). At Hobart, all samples were maintained at $-80^{\circ} \mathrm{C}$.

Electrophoresis. Extracts of longitudinal muscle were prepared by grinding small tissue fragments in 2 drops of homogenising solution in a $1.5 \mathrm{ml}$ microcentrifuge tube and spinning at $10000 \times g$ for $2 \mathrm{~min}$. The homogenising solution was made up of $100 \mathrm{ml}$ distilled water, $10 \mathrm{mg}$ NADP and $100 \mu \mathrm{l}$ of $\beta$-mercaptoethanol. Samples were maintained at $4^{\circ} \mathrm{C}$ during preparation.
The supernatant was used for electrophoresis. All electrophoresis runs used Helena Titan III cellulose acetate plates. Eighteen enzyme systems, representing 23 loci, were examined either with a $200 \mathrm{~V}$ tris-glycine (Hebert \& Beaton 1989) or $150 \mathrm{~V}$ tris-citrate $(75 \mathrm{mM}$ tris, $25 \mathrm{mM}$ citric acid, $\mathrm{pH} 7.0$ ) buffer system (Table 2). Standard staining procedures were followed (Richardson et al. 1986, Hebert \& Beaton 1989). When an enzyme was encoded by 2 loci, the more anodally migrating enzyme was suffixed as 1 . Alleles were numbered

Table 2. Sabella spallanzanii and Sabella pavonina. Enzymes, loci, electrophoresis conditions, and likely subunit numbers as assessed from heterozygote banding patterns. Buffer A: tris-glycine; buffer B: tris-citrate

\begin{tabular}{|c|c|c|c|c|c|}
\hline Enzyme & EC number & Locus & Buffer & Run time (min) & Subunit number \\
\hline Aspartate aminotransferase & 2.6 .1 .1 & Aat-1, Aat-2 & $\mathrm{B}$ & 75 & 2 \\
\hline Aconitase & 4.2 .1 .3 & Acon & $\mathrm{B}$ & 60 & 1 \\
\hline Arginine phosphokinase & 2.7 .3 .3 & $A p k$ & $B$ & 100 & 4 \\
\hline Esterase D & 3.1.1.- & EstD-1, EstD-2 & A & 20 & $2^{\circ}$ \\
\hline Glyceraldehyde-3-phosphate dehydrogenase & 1.2 .1 .12 & G3pdh & $\mathrm{B}$ & 75 & 4 \\
\hline Isocitrate dehydrogenase & 1.1 .1 .42 & $I d h$ & $\mathrm{~B}$ & 75 & 2 \\
\hline Lactate dehydrogenase & 1.1 .1 .27 & $L d h$ & A & 20 & invariant \\
\hline Malate dehydrogenase & 1.1 .1 .37 & Mdh & $\mathrm{B}$ & 90 & 2 \\
\hline Malic enzyme & 1.1 .1 .40 & $\mathrm{Me}-1, \mathrm{Me}-2$ & $\mathrm{~A}$ & 30 & uncertain \\
\hline Mannosephosphate isomerase & 5.3 .1 .8 & Mpi & A & 25 & 1 \\
\hline Peptidase (valine-leucine) & 3.4 .11 - & Pep-vl-1, Pep-vl-2 & $\mathrm{B}$ & 65 & $2^{b}$ \\
\hline Peptidase (leucine-glycine-glycine) & $3.4 .11 .-$ & Pep-lgg & $\mathrm{B}$ & 75 & 1 \\
\hline Peptidase (phenylalanine-proline) & $3.4 .11 .-$ & Pep-pp & A & 100 & 2 \\
\hline 6-Phosphogluconate dehydrogenase & 1.1 .1 .44 & $6 P g d h$ & $\mathrm{~B}$ & 55 & 2 \\
\hline Glucosephosphate isomerase & 5.3 .1 .9 & Pgi & A & 30 & 2 \\
\hline Phosphoglucomutase & 5.4 .2 .2 & Pgm-1, Pgm-2 & A & 28 & 1 \\
\hline Sorbitol (iditol) dehydrogenase & 1.1 .1 .14 & Sdh & A & 30 & invariant \\
\hline Xanthine dehydrogenase & 1.2 .3 .2 & $X d h$ & A & 35 & 2 \\
\hline $\begin{array}{l}\text { EstD- } 2 \text { is a dimer, EstD- } 1 \text { is invariant } \\
{ }^{2} P e p-v l-1 \text { is a dimer, Pep-vl-2 is invarlant }\end{array}$ & & & & & \\
\hline
\end{tabular}


according to the mobilities of their products relative to that of the most common allele in the Victoria sample, numbered 100 .

Statistical tests. Tests were carried out to examine whether the observed genotype numbers were in accord with those expected in a sexually reproducing randomly mating population. Observed and HardyWeinberg expected numbers were compared with chisquared tests. Tests were only considered reliable if all genotype classes in a test had an expected value of 1 or greater. This frequently necessitated pooling rarer alleles.

The degree of genetic diversity among collections was analysed in 3 ways: (1) Contingency chi-squared tests were used to determine whether allele frequencies of variable loci were homogeneous over collections. The Monte Carlo randomisation approach of Roff \& Bentzen (1989) was used. This eliminates the need to pool rare alleles. One thousand randomisations were carried out for each test. (2) Nei's (1973) gene diversity $\left(G_{S T}\right)$ statistics were used to quantify the extent of differentiation among collections. The $G_{S T}$ value represenls the proportion of genetic diversity that can be attributed to differences between collections. A bootstrapping procedure with 1000 replicates was used to estimate the magnitude of $G_{S T}$ that could be attributed to sampling error alone and to assess the statistical significance of the observed value. (3) Genetic distances between collections were assessed in 2 ways. The first used Nei's (1978) unbiased genetic distance measure. The resulting genetic distance matrix was converted to a dendrogram by cluster analysis with the UPGMA (unweighted pairgroup method with averaging) algorithm. The second used Rogers' (1972) genetic distance method, and the derived dendrogram is a Wagner tree rooted by the out-group (Sabella pavonina).

In all analyses involving multiple tests, the Bonferroni technique was used to adjust significance levels. The predetermined significance level for the experiment, $\alpha$, of 0.05 was divided by the number of tests to obtain a corrected significance level of $\alpha$.

\section{RESULTS}

Enzymes encoded by a total of 23 loci provided clear and reproducible results. Subunit numbers (Table 2), with 1 exception, were as listed in Ward et al. (1992). The exception was Apk which Ward et al. (1992) consider is a monomer in arthropods and molluscs, but a dimer in annelids and echinoderms. Sabella is an annelid, but banding patterns for the Apk*115/100 heterozygote were clearly 5-banded, indicating a tetramer. The much more common heterozygote
Apk $100 / 95$ gave a broad band of activity, presumably because the 5 bands were too close together to separate adequately.

The 2 Alicante collections, one from a polluted site and the other from an unpolluted site, showed differences in allele frequencies (data not shown) at 1 of 16 variable loci. This was Pep-lgg $(\mathrm{p}=0.016)$, but after Bonferroni correction for multiple tests, the differences were not statistically significant ( $\alpha$ altered from 0.05 to $0.05 / 16=0.003$ ). Thus these 2 collections were pooled in all subsequent analyses

The 2 collections from the south of France (Carteau and Marseille) were separated by a greater geographic distance than the 2 Alicante collections and showed significant differences in gene frequencies at one of the 18 variable loci $(M e-1, p<0.001, \alpha=0.05 / 18=$ $0.003)$. These 2 collections were kept separate in all analyses.

\section{Sabella spallanzanii and S. pavonina: levels of genetic variability}

Genotype distributions within the collections of Sabella spallanzanii showed good agreement with Hardy-Weinberg expectations. There were 66 valid tests, but only 2 showed probabilities of agreement less than 0.05. These were pep-vl-1 in South Australia ( $\mathrm{p}=$ $0.0006)$, and Pgi in Carteau ( $p=0.024)$. Corrections of $\alpha$ levels for 66 tests reduced $\alpha$ to 0.0008 , so the $P g i$ result becomes non-significant while the $P e p-v-1$ result (with a heterozygote deficiency: 30 heterozygotes observed, 45.8 expected) remains statistically significant. However, whether this deviation represents a real biological phenomenon or simply a chance sampling artefact is uncertain; repeat sampling from that population would be necessary to discriminate between these possibilities. The $10 \mathrm{~S}$. pavonina sampled were not tested for conformance to Hardy-Weinberg expectations.

Sabella spallanzanii showed abundant genetic variation (Table 3). Only 5 loci were monomorphic in all collections (EstD-1,Ldh, Me-2, Pep-vl-2, Sdh). The percentage of polymorphic loci ranged from about 45 to $60 \%$ (0.95 criterion) or 50 to $80 \%$ ( 0.99 criterion), and the mean expected heterozygosity per locus ranged from about 0.20 to 0.27 (Table 4)

The Australian collections were clearly less variable, over all loci, than the European collections (Table 4). Mean numbers of alleles per locus were around 2.0 in the Australian collections, compared with between 2.0 and 2.8 in the European collections. The average sample size of the European collections was slightly greater than half that of the Australian collections; had sizes been closer, it is likely that this difference would 
Table 3. Sabella spallanzanii and S. pavonina. Allele frequencies, sample sizes (n: number of individuals), and locus heterozygosities (het.. Hardy-Weinberg expected heterozygosity)

\begin{tabular}{|c|c|c|c|c|c|c|c|c|c|}
\hline \multirow[t]{3}{*}{ Locus } & \multirow[t]{3}{*}{ Allele } & \multirow{3}{*}{ Victoria } & \multicolumn{6}{|c|}{ Sabella spallanzanii } & \multirow{3}{*}{$\begin{array}{c}\text { Sabella } \\
\text { pavonina }\end{array}$} \\
\hline & & & South & Western & Atlantic & & Mediterranean & & \\
\hline & & & Australia & Australia & Roscoff & Carteau & Marseille & Alicante & \\
\hline \multirow[t]{7}{*}{ Aat-1 } & 110 & 0 & 0 & 0 & 0.008 & 0.010 & 0 & 0 & 0 \\
\hline & 100 & 0.949 & 0.960 & 0.900 & 0.992 & 0.979 & 1 & 0.992 & 0 \\
\hline & 95 & 0 & 0 & 0 & 0 & 0 & 0 & 0 & 0.050 \\
\hline & 93 & 0 & 0 & 0 & 0 & 0 & 0 & 0 & 0.900 \\
\hline & 90 & 0.051 & 0.040 & 0.100 & 0 & 0.010 & 0 & 0.008 & 0.050 \\
\hline & $\mathrm{n}$ & 108 & 100 & 100 & 62 & 48 & 50 & 60 & 10 \\
\hline & het. & 0.097 & 0.077 & 0.180 & 0.016 & 0.041 & 0 & 0.016 & 0.185 \\
\hline \multirow[t]{5}{*}{ Aat-2 } & 110 & 0 & 0 & 0 & 0 & 0 & 0 & 0 & 0.250 \\
\hline & 105 & 0 & 0 & 0 & 0 & 0.031 & 0.010 & 0.042 & 0.650 \\
\hline & 100 & 1 & 1 & 1 & 1 & 0.969 & 0.990 & 0.958 & 0.100 \\
\hline & $\mathrm{n}$ & 108 & 100 & 100 & 62 & 48 & 50 & 60 & 10 \\
\hline & het. & 0 & 0 & 0 & 0 & 0.06 & 0.02 & 0.081 & 0.505 \\
\hline \multirow[t]{5}{*}{ Acon } & 105 & 0.153 & 0.245 & 0.180 & 0.097 & 0.083 & 0.070 & 0.075 & 0 \\
\hline & 100 & 0.792 & 0.680 & 0.725 & 0.476 & 0.604 & 0.690 & 0.800 & 1 \\
\hline & 95 & 0.056 & 0.075 & 0.095 & 0.427 & 0.313 & 0.240 & 0.125 & 0 \\
\hline & $\mathrm{n}$ & 108 & 100 & 100 & 62 & 48 & 50 & 60 & 10 \\
\hline & het. & 0.346 & 0.472 & 0.433 & 0.582 & 0.530 & 0.461 & 0.339 & 0 \\
\hline \multirow[t]{5}{*}{ Apk } & 115 & 0 & 0 & 0 & 0 & 0 & 0.020 & 0.025 & 0 \\
\hline & 100 & 0.741 & 0.725 & 0.790 & 1 & 0.958 & 0.940 & 0.867 & 0 \\
\hline & 95 & 0.259 & 0.275 & 0.210 & 0 & 0.042 & 0.040 & 0.108 & 1 \\
\hline & $\mathrm{n}$ & 108 & 100 & 100 & 62 & 48 & 50 & 60 & 10 \\
\hline & het. & 0.384 & 0.399 & 0.332 & 0 & 0.081 & 0.114 & 0.236 & 0 \\
\hline \multirow[t]{3}{*}{ EstD-1 } & 100 & 1 & 1 & 1 & 1 & 1 & 1 & 1 & 1 \\
\hline & $\mathrm{n}$ & 108 & 100 & 100 & 62 & 48 & 50 & 60 & 10 \\
\hline & het. & 0 & 0 & 0 & 0 & 0 & 0 & 0 & 0 \\
\hline \multirow[t]{5}{*}{ EstD-2 } & 115 & 0 & 0 & 0 & 0.032 & 0.149 & 0.130 & 0.083 & 0 \\
\hline & 105 & 0 & 0 & 0 & 0 & 0 & 0 & 0 & 1 \\
\hline & 100 & 1 & 1 & 1 & 0.968 & 0.851 & 0.870 & 0.917 & 0 \\
\hline & $\mathrm{n}$ & 108 & 100 & 100 & 62 & 47 & 50 & 60 & 10 \\
\hline & het. & 0 & 0 & 0 & 0.062 & 0.254 & 0.226 & 0.152 & 0 \\
\hline \multirow[t]{8}{*}{ G3pdh } & 100 & 0.481 & 0.420 & 0.425 & 0.766 & 0.833 & 0.910 & 0.617 & 0 \\
\hline & 80 & 0 & 0 & 0 & 0 & 0.042 & 0.030 & 0.150 & 0 \\
\hline & 70 & 0 & 0 & 0 & 0 & 0 & 0 & 0 & 0.950 \\
\hline & 60 & 0.051 & 0.105 & 0.105 & 0 & 0.031 & 0 & 0.017 & 0 \\
\hline & 50 & 0 & 0 & 0 & 0 & 0 & 0 & 0 & 0.050 \\
\hline & 40 & 0.468 & 0.475 & 0.470 & 0.234 & 0.094 & 0.060 & 0.217 & 0 \\
\hline & $\mathrm{n}$ & 108 & 100 & 100 & 62 & 48 & 50 & 60 & 10 \\
\hline & het. & 0.547 & 0.587 & 0.588 & 0.359 & 0.295 & 0.167 & 0.549 & 0.095 \\
\hline \multirow[t]{4}{*}{$I d h$} & 100 & 0.977 & 1 & 0.980 & 1 & 0.948 & 0.960 & 0.950 & 1 \\
\hline & 95 & 0.023 & 0 & 0.020 & 0 & 0.052 & 0.040 & 0.050 & 0 \\
\hline & $\mathrm{n}$ & 108 & 100 & 100 & 62 & 48 & 50 & 60 & 10 \\
\hline & het. & 0.045 & 0 & 0.039 & 0 & 0.099 & 0.077 & 0.095 & 0 \\
\hline \multirow[t]{3}{*}{$L d h$} & 100 & 1 & 1 & 1 & 1 & 1 & 1 & 1 & 1 \\
\hline & $\mathrm{n}$ & 108 & 100 & 100 & 62 & 48 & 50 & 60 & 2 \\
\hline & het. & 0 & 0 & 0 & 0 & 0 & 0 & 0 & 0 \\
\hline \multirow[t]{4}{*}{$M d h$} & 115 & 0.093 & 0.040 & 0.040 & 0 & 0.010 & 0.010 & 0.033 & 0 \\
\hline & 100 & 0.907 & 0.960 & 0.960 & 1 & 0.990 & 0.990 & 0.967 & 1 \\
\hline & $\mathrm{n}$ & 108 & 100 & 100 & 62 & 48 & 50 & 60 & 10 \\
\hline & het. & 0.169 & 0.077 & 0.077 & 0 & 0.020 & 0.020 & 0.064 & 0 \\
\hline Me- 1 & 105 & 0.014 & 0 & 0 & 0 & 0.042 & 0.170 & 0.008 & 0 \\
\hline & 100 & 0.986 & 1 & 1 & 1 & 0.833 & 0.810 & 0.933 & 0 \\
\hline & 95 & 0 & 0 & 0 & 0 & 0.125 & 0.020 & 0.058 & 0 \\
\hline & 90 & 0 & 0 & 0 & 0 & 0 & 0 & 0 & 1 \\
\hline & $\mathrm{n}$ & 108 & 100 & 100 & 62 & 48 & 50 & 60 & 10 \\
\hline & het. & 0.028 & 0 & 0 & 0 & 0.289 & 0.315 & 0.126 & 0 \\
\hline
\end{tabular}


Table 3. (continued)

\begin{tabular}{|c|c|c|c|c|c|c|c|c|c|}
\hline \multirow[t]{3}{*}{ Locus } & \multirow[t]{3}{*}{ Allele } & \multirow{3}{*}{ Victoria } & \multicolumn{6}{|c|}{ Sabella spallanzamii } & \multirow{3}{*}{$\begin{array}{c}\text { Sabella } \\
\text { pavonina }\end{array}$} \\
\hline & & & South & Western & Atlantic & & Mediterranean & & \\
\hline & & & Australia & Australia & Roscoff & Carteau & Ma:seille & Alicante & \\
\hline \multirow[t]{3}{*}{$\mathrm{Me}-2$} & 100 & 1 & 1 & 1 & 1 & 1 & 1 & 1 & 1 \\
\hline & $\mathrm{n}$ & 108 & 100 & 100 & 62 & 48 & 50 & 60 & 10 \\
\hline & het. & 0 & 0 & 0 & 0 & 0 & 0 & 0 & 0 \\
\hline \multirow[t]{7}{*}{$M p i$} & 108 & 0 & 0 & 0 & 0 & 0 & 0 & 0 & 0.050 \\
\hline & 105 & 0 & 0 & 0 & 0 & 0.031 & 0.040 & 0 & 0 \\
\hline & 103 & 0 & 0 & 0 & 0 & 0 & 0 & 0 & 0.950 \\
\hline & 100 & 0.519 & 0.620 & 0.480 & 0.427 & 0.583 & 0.540 & 0.583 & 0 \\
\hline & 95 & 0.481 & 0.380 & 0.520 & 0.573 & 0.333 & 0.410 & 0.408 & 0 \\
\hline & 90 & 0 & 0 & 0 & 0 & 0.052 & 0.010 & 0.008 & 0 \\
\hline & $\begin{array}{c}\mathrm{n} \\
\text { het. }\end{array}$ & $\begin{array}{c}108 \\
0.499\end{array}$ & $\begin{array}{c}100 \\
0.471\end{array}$ & $\begin{array}{c}100 \\
0.499\end{array}$ & $\begin{array}{c}62 \\
0.489\end{array}$ & $\begin{array}{c}48 \\
0.546\end{array}$ & $\begin{array}{c}50 \\
0.539\end{array}$ & $\begin{array}{c}60 \\
0.494\end{array}$ & $\begin{array}{c}10 \\
0.095\end{array}$ \\
\hline \multirow[t]{6}{*}{$P e p-v l-I$} & 110 & 0 & 0 & 0 & 0.210 & 0 & 0 & 0.017 & 0 \\
\hline & 100 & 0.767 & 0.633 & 0.710 & 0.323 & 0.771 & 0.796 & 0.808 & 0 \\
\hline & 90 & 0.233 & 0.367 & 0.290 & 0.468 & 0.229 & 0.204 & 0.175 & 0 \\
\hline & 75 & 0 & 0 & 0 & 0 & 0 & 0 & 0 & 0.250 \\
\hline & 70 & 0 & 0 & 0 & 0 & 0 & 0 & 0 & 0.750 \\
\hline & n & 105 & 98 & 100 & 62 & 48 & $\begin{array}{c}49 \\
0325\end{array}$ & $\begin{array}{l}60 \\
0.316\end{array}$ & $\begin{array}{c}10 \\
0375\end{array}$ \\
\hline \multirow{5}{*}{ Pep-vl-2 } & 130 & 0 & 0 & 0 & 0 & 0 & 0 & 0 & 0.200 \\
\hline & 115 & 0 & 0 & 0 & 0 & 0 & 0 & 0 & 0.800 \\
\hline & 100 & 1 & 1 & 1 & 1 & 1 & 1 & 1 & 0 \\
\hline & $\mathrm{n}$ & 108 & 100 & 100 & 62 & 48 & 50 & 60 & 10 \\
\hline & het. & 0 & 0 & 0 & 0 & 0 & 0 & 0 & 0.320 \\
\hline \multirow[t]{9}{*}{ Pep-lgg } & 145 & 0 & 0 & 0 & 0 & 0 & 0 & 0 & 0.750 \\
\hline & 135 & 0 & 0 & 0 & 0 & 0 & 0 & 0 & 0.050 \\
\hline & 125 & 0 & 0 & 0 & 0 & 0 & 0 & 0 & 0.200 \\
\hline & 110 & 0.329 & 0.405 & 0.380 & 0 & 0.011 & 0 & 0 & 0 \\
\hline & 100 & 0.583 & 0.535 & 0.560 & 0.185 & 0.383 & 0.470 & 0.475 & 0 \\
\hline & 90 & 0.088 & 0.060 & 0.060 & 0.815 & 0.585 & 0480 & 0.500 & 0 \\
\hline & 80 & 0 & 0 & 0 & 0 & 0.021 & 0050 & 0.025 & 0 \\
\hline & $n$ & 108 & 100 & $\begin{array}{c}100 \\
0538\end{array}$ & $\begin{array}{c}62 \\
302\end{array}$ & 47 & $\begin{array}{c}50 \\
0546\end{array}$ & $\begin{array}{c}60 \\
0524\end{array}$ & $\begin{array}{c}10 \\
0395\end{array}$ \\
\hline & & & & & 0.302 & 0.511 & & & 0 \\
\hline \multirow[t]{5}{*}{$P e p-p p$} & $\begin{array}{l}115 \\
110\end{array}$ & $\begin{array}{l}0.088 \\
0.259\end{array}$ & $\begin{array}{l}0.105 \\
0.170\end{array}$ & $\begin{array}{l}0.120 \\
0.265\end{array}$ & $\begin{array}{l}0.210 \\
0.274\end{array}$ & $\begin{array}{l}0.188 \\
0.365\end{array}$ & $\begin{array}{l}0.245 \\
0.298\end{array}$ & $\begin{array}{l}0.217 \\
0.392\end{array}$ & $\begin{array}{l}0 \\
0\end{array}$ \\
\hline & 100 & 0.426 & 0.550 & 0.355 & 0.444 & 0.302 & 0372 & 0.283 & 0.500 \\
\hline & 90 & 0.227 & 0.175 & 0.260 & 0.065 & 0.125 & 0074 & 0.108 & 0.500 \\
\hline & 80 & 0 & 0 & 0 & 0.008 & 0.021 & 0011 & 0 & 0 \\
\hline & $\mathrm{n}$ & 108 & 100 & 100 & 62 & 48 & $\begin{array}{c}47 \\
0707\end{array}$ & $\begin{array}{c}60 \\
0708\end{array}$ & $\begin{array}{c}8 \\
0500\end{array}$ \\
\hline \multirow{7}{*}{$6 P g d h$} & 105 & 0 & 0 & 0 & 0 & 0.010 & 0010 & 0.017 & 0.050 \\
\hline & 100 & 0.903 & 0.910 & 0.905 & 0.798 & 0.792 & 0.830 & 0.775 & 0.400 \\
\hline & 98 & 0 & 0 & 0 & 0 & 0 & 0 & 0 & 0.500 \\
\hline & 95 & 0.097 & 0.090 & 0.095 & 0.202 & 0.198 & 0150 & 0.200 & 0 \\
\hline & 90 & 0 & 0 & 0 & 0 & 0 & 0.010 & 0.008 & 0.050 \\
\hline & $\mathrm{n}$ & 108 & 100 & 100 & 62 & 48 & 50 & 60 & 10 \\
\hline & het. & 0.175 & 0.164 & 0.172 & 0.322 & 0.333 & 0288 & 0.359 & 0.585 \\
\hline \multirow[t]{6}{*}{$P g m-1$} & 105 & 0.074 & 0.070 & 0.050 & 0 & 0 & 0.010 & 0.008 & 0 \\
\hline & 100 & 0.926 & 0.930 & 0.950 & 1 & 0.979 & 0.970 & 0.983 & 0.050 \\
\hline & 30 & 0 & 0 & 0 & 0 & 0.021 & 0.020 & 0.008 & 0.850 \\
\hline & 85 & 0 & 0 & 0 & 0 & 0 & 0 & 0 & 0.100 \\
\hline & $\mathrm{n}$ & 108 & 100 & 100 & 62 & 48 & 50 & 60 & 10 \\
\hline & het. & 0.137 & 0.130 & 0.095 & 0 & 0.041 & 0.059 & 0.034 & 0.265 \\
\hline \multirow[t]{6}{*}{ Pgm-2 } & 110 & 0 & 0 & 0 & 0.040 & 0.277 & 0.316 & 0.083 & 0.750 \\
\hline & 100 & 1 & 1 & 1 & 0.524 & 0.596 & 0.602 & 0.817 & 0 \\
\hline & 90 & 0 & 0 & 0 & 0.371 & 0.117 & 0.061 & 0.067 & 0.250 \\
\hline & 35 & 0 & 0 & 0 & 0.065 & 0.011 & 0.020 & 0.033 & 0 \\
\hline & $\mathrm{n}$ & 108 & 100 & 100 & 62 & 47 & 49 & 60 & 10 \\
\hline & ret. & 0 & 0 & 0 & 0.582 & 0.554 & 0.534 & 0.320 & 0.375 \\
\hline
\end{tabular}


Table 3. (continued)

\begin{tabular}{|c|c|c|c|c|c|c|c|c|c|}
\hline \multirow[t]{3}{*}{ Locus } & \multirow[t]{3}{*}{ Allele } & \multicolumn{7}{|c|}{ Sabella spallanzanii } & \multirow{3}{*}{$\begin{array}{c}\text { Sabella } \\
\text { pavonina }\end{array}$} \\
\hline & & Victoria & South & Western & Atlantic & & Mediterranean & & \\
\hline & & & Australia & Australia & Roscoff & Carteau & Marseille & Alicante & \\
\hline \multirow[t]{10}{*}{ Pgi } & 135 & 0 & 0 & 0 & 0.121 & 0.031 & 0.010 & 0.033 & 0 \\
\hline & 125 & 0 & 0 & 0 & 0.097 & 0.229 & 0.160 & 0.217 & 0 \\
\hline & 115 & 0 & 0 & 0 & 0.016 & 0.083 & 0.030 & 0.042 & 0 \\
\hline & 100 & 0.954 & 0.945 & 0.945 & 0.226 & 0.500 & 0.630 & 0.467 & 0 \\
\hline & 95 & 0 & 0 & 0 & 0 & 0 & 0 & 0.025 & 0.500 \\
\hline & 90 & 0 & 0 & 0 & 0 & 0.052 & 0.040 & 0.050 & 0 \\
\hline & 80 & 0.046 & 0.055 & 0.055 & 0.540 & 0.104 & 0.130 & 0.158 & 0.300 \\
\hline & 70 & 0 & 0 & 0 & 0 & 0 & 0 & 0.008 & 0.200 \\
\hline & $\mathrm{n}$ & 108 & 100 & 100 & 62 & 48 & 50 & 60 & 10 \\
\hline & het. & 0.088 & 0.104 & 0.104 & 0.633 & 0.676 & 0.558 & 0.704 & 0.620 \\
\hline \multirow[t]{4}{*}{$S d h$} & 115 & 0 & 0 & 0 & 0 & 0 & 0 & 0 & 1 \\
\hline & 100 & 1 & 1 & 1 & 1 & 1 & 1 & 1 & 0 \\
\hline & $\mathrm{n}$ & 108 & 100 & 100 & 62 & 48 & 50 & 60 & 10 \\
\hline & het. & 0 & 0 & 0 & 0 & 0 & 0 & 0 & 0 \\
\hline \multirow[t]{8}{*}{$X d h$} & 125 & 0 & 0 & 0 & 0 & 0 & 0 & 0 & 1 \\
\hline & 110 & 0 & 0 & 0 & 0.145 & 0 & 0.040 & 0.045 & 0 \\
\hline & 105 & 0.079 & 0.060 & 0.155 & 0.306 & 0.188 & 0.250 & 0.295 & 0 \\
\hline & 100 & 0.611 & 0.600 & 0.555 & 0.532 & 0.438 & 0.420 & 0.295 & 0 \\
\hline & 85 & 0.269 & 0.305 & 0.245 & 0.016 & 0.250 & 0.240 & 0.205 & 0 \\
\hline & 80 & 0.042 & 0.035 & 0.045 & 0 & 0.125 & 0.050 & 0.161 & 0 \\
\hline & $\mathrm{n}$ & 108 & 100 & 100 & 62 & 48 & 50 & 56 & 10 \\
\hline & het. & 0.546 & 0.542 & 0.606 & 0.602 & 0.695 & 0.699 & 0.756 & 0 \\
\hline
\end{tabular}

Table 4. Sabella spallanzanii and S. pavonina. Summary of genetic variability in all collections ( \pm SE) for 23 loci. There are 2 possible definitions of polymorphism: one where the most common allele has a frequency $\leq 0.95$, and one where the most common allele has a frequency $\leq 0.99$; the $\leq 0.95$ criterion is shown here. HW: Hardy-Weinberg

\begin{tabular}{|c|c|c|c|c|}
\hline Collection & $\begin{array}{c}\text { Mean sample size } \\
\text { per locus }\end{array}$ & $\begin{array}{c}\text { Mean no. of alleles } \\
\text { per locus }\end{array}$ & $\begin{array}{l}\text { Percentage of loci poly- } \\
\text { morphic }\{0.95 \text { criterion }\}\end{array}$ & $\begin{array}{c}\text { Mean HW expected hetero- } \\
\text { zygosity per locus }\end{array}$ \\
\hline \multicolumn{5}{|l|}{ S. spallanzanii } \\
\hline Victoria & $107.9 \pm 0.1$ & $2.0 \pm 0.2$ & 52.17 & $0.203 \pm 0.048$ \\
\hline South Australia & $99.9 \pm 0.1$ & $1.9 \pm 0.2$ & 47.83 & $0.204 \pm 0.050$ \\
\hline Western Australia & $100.0 \pm 0.0$ & $1.9 \pm 0.2$ & 52.17 & $0.210 \pm 0.051$ \\
\hline Roscoff & $62.0 \pm 0.0$ & $2.0 \pm 0.3$ & 43.48 & $0.231 \pm 0.058$ \\
\hline Carteau & $47.9 \pm 0.1$ & $2.7 \pm 0.3$ & 56.52 & $0.268 \pm 0.055$ \\
\hline Marseille & $49.8 \pm 0.1$ & $2.7 \pm 0.3$ & 56.52 & $0.248 \pm 0.053$ \\
\hline Alicante & $59.8 \pm 0.2$ & $2.8 \pm 0.3$ & 60.87 & $0.257 \pm 0.054$ \\
\hline \multicolumn{5}{|l|}{ S. pavonina } \\
\hline Roscoff & $9.8 \pm 0.2$ & $1.8 \pm 0.2$ & 52.17 & $0.198 \pm 0.049$ \\
\hline
\end{tabular}

have been accentuated. The percentage of loci polymorphic in the Australian collections ranged from 47.8 to 52.2 ( 0.95 criterion) and 56.5 to 65.2 ( 0.99 criterion), values again generally less than in the European collections: 43.5 to $60.9(0.95)$ and 47.8 to $78.3(0.99)$.

The best single parameter for comparing variation is mean expected heterozygosity per locus $(H)$, as it is far less dependent on sample size than either mean numbers of alleles per locus or mean percentage of loci polymorphic. $H$ for the Australian collections ranged narrowly from 0.203 to 0.210 ; for the overseas collections it had a broader range of 0.231 to 0.268 . The mean $H$ of the Australian collections was 0.206 , about $18 \%$ less than the mean $H$ of the 4 European collections $(0.251)$. This loss of overall variation is by no means distributed uniformly over all loci. Indeed some loci (Aat-1, Apk, G3pdh, Mdh, Pgm-1) show more variation in the Australian collections. However, this is more than offset by those loci that are more variable in Europe (EstD-2, Me-1, 6Pgdh, Pgm-2, Pgi, Xdh). Particularly striking losses of variation in Australia are seen in Pgm-2 and Pgi. Pgm-2 is monomorphic in Australian collections but highly polymorphic in all European collections tested (heterozygosities ranging from 
Table 5. Sabclla spallanzanii. Pairwise collection comparisons for unique alleles. Numbers are numbers of alleles present in the 'column' population that are not present in the 'row' population

\begin{tabular}{|c|c|c|c|c|}
\hline & \multicolumn{4}{|c|}{ Number of unique alleles } \\
\hline & Roscoff & Carteau & Marseille & Alicante \\
\hline Roscoff & & 17 & 17 & 20 \\
\hline Carteau & 2 & & 4 & 6 \\
\hline Marseille & 2 & 4 & & 5 \\
\hline Alicante & 2 & 4 & 2 & \\
\hline
\end{tabular}

0.320 to 0.582). Pgi shows little variation in Australian collections (heterozygosities ranging from 0.088 to 0.104 ), but is strikingly variable in European collections (heterozygosities ranging from 0.558 to 0.704 ).

Among the European collections, the Mediterranean collections showed more variation than the Atlantic collection (Table 4). This was true for all measures of variation. mean numbers of alleles (2.7 to 2.8 vs 2.0 ), percentage of loci polymorphic $(0.95$ criterion, 57 to $61 \%$ vs $43 \%$; 0.99 criterion, 74 to $78 \%$ vs $48 \%$ ), and expected heterozygosity $(0.25$ to 0.27 vs 0.23$)$. The Atlantic collection has 2 alleles not found in Carteau, 2 alleles not found in Marseille, and 2 alleles not found in Alicante (Table 5). On the other hand, Carteau, Marseille and Alicante have, respectively, 17, 17 and 20 alleles not found at Roscoff

Sabella pavonina showed slightly less variability than European S. spallanzanii, in terms of average numbers of alleles per locus and expected heterozygosity (Table 4). However, while the same 23 loci were examined in $S$. pavonina, only about 10 individuals were scored. Thus it is not unexpected that fewer alleles per locus were detected in S. pavonina: with a sample size closer to the 50 or so of each of the European $S$. spallanzanii, it is likely that more alleles would have been found.

\section{Sabella spallanzanii: genetic relationships among Australian populations}

Fifteen loci were variable in the Australian collections. While some of the data were suggestive of limited differentiation the estimated probabilities of no significant differentiation, using Roff \& Bentzen's (1989) Monte-Carlo chi-squared test, in descending order, were: 0.985, 6Pgdh; 0.915, Pgi; 0.641, Pgm-1; 0.449, Pep-Igg; 0.286, Apk; 0.203, G3pdh; 0.151, Idh; $0.104, \mathrm{Me}-1 ; 0.063$, Acon $_{i} 0.042, X d h_{i}$ 0.032, Mdh; 0.029, Aat-1; 0.012, Mpi; 0.007, Pep-vl-1; 0.006, Pep$p p]$, after carrying out Bonferroni adjustments of $\alpha$ levels for multiple tests ( $\alpha$ changes from 0.05 to $0.05 / 15=$
0.003), none was statistically significant. This conclusion still holds after carrying out the less conservative sequential Bonferroni test (Hochberg 1988). Clearly the 3 Australian collections, all with a sample size of around 100, are very similar to one another.

\section{Sabella spallanzanii: genetic relationships among European populations}

There were 18 variable loci in the European collections. Of these, 8 (Aat-1, Aat-2, EstD-2, Idh, Mdh, $6 P g d h, P e p-p p, P g m-1)$ showed no significant differentiation (probabilities respectively $0.843,0.546,0.528$, $0.256,0.236,0.078,0.057,0.012$, with $\alpha=0.05 / 18=$ 0.003 ) and 10 (Acon, Apk, G3pdh, Me-1, Mpi, Pep-lgg, Pep-vl, Pgi, Pgm-2, Xdh) showed significant differentiation (all with $\mathrm{p}<0.001$ ). So clearly there is significant differentiation among the Europcan collections.

For each of the 10 differentiated loci, the 4 collections were compared pairwise to determine the source(s) of the differentiation. The Carteau and Marseille collections, which were only about $40 \mathrm{~km}$ apart (H. Zibrowius pers comm.), were significantly different for only 1 out of the 10 loci, Me-1 $(p<0.001)$. The Carteau and Marseille collections were each significantly differentiated from the remaining Mediterranean collection, Alicante, by 3 of the 10 loci (Carteau: Acon, G3pdh, Pgm-2; Marseille: G3pdh, Me1. Pgm-2; all $\mathrm{p}<0.001$ ). The Atlantic Roscoff collection, on the other hand, was well differentiated from all 3 Mediterranean collections for between 8 and all 10 loci (p ranging from 0.004 to $<0.001$ ). Clearly, the Mediterranean collections are genetically more similar to one another than they are to the Atlantic collection.

\section{Sabella spallanzanii: genetic relationships between European and Australian populations}

There was extensive spatial differentiation in allele frequencies among collections, with 17 of the 18 variable loci showing significant allele frequency heterogeneity after chi-squared analysis of allele frequencies (Table 6). The only variable locus not to show such heterogeneity (following Bonferroni correction to $\alpha$ values, giving $\alpha=0.05 / 18=0.0028$ ) was $l d h$.

The extent of the heterogeneity was quantified for each locus by a $G_{S T}$ analysis (Table 6). Here, the observed extent of differentiation among populations $\left(G_{S T}\right)$ was compared with sampling error, i.e. the extent of $G_{S T}$ expected in those sample sizes from a panmictic population $\left(G_{S T_{\text {null }}}\right)$. Of the 18 variable loci, only Idh (as in the chi-squared analysis) failed to show more between-collection differentiation than expected by 
Table 6. Sabella spallanzanii. Chi-squared and $G_{S T}$ analyses of the extent of differentiation among collections. H is the total heterozygosity across all populations, $H_{S}$ is the average heterozygosity per collection, $G_{S T}$ is the proportion of the variation attributable to collection differences, $G_{S T \cdot n u l l}$ is the expected value of $G_{S T}$ that can be attributed to sampling error alone

\begin{tabular}{|c|c|c|c|c|c|c|c|}
\hline Locus & $H_{T}$ & $H_{S}$ & Chi-squared & $\mathrm{p}$ & $G_{S T}$ & Mean $G_{S r \cdot \text { rull }}$ & $\mathrm{p}$ \\
\hline Aat -1 & 0.066 & 0.063 & 42.661 & $<0.001$ & 0.033 & 0.006 & $<0.001$ \\
\hline Aat-2 & 0.023 & 0.023 & 27.855 & $<0.001$ & 0.022 & 0.006 & 0.002 \\
\hline Acon & 0.484 & 0.452 & 146.614 & $<0.001$ & 0.065 & 0.007 & $<0.001$ \\
\hline Apk & 0.242 & 0.221 & 102.234 & $<0.001$ & 0.089 & 0.006 & $<0.001$ \\
\hline EstD-1 & 0 & 0 & - & - & - & - & - \\
\hline EstD-2 & 0.106 & 0.099 & 84.057 & $<0.001$ & 0.067 & 0.007 & $<0.001$ \\
\hline$G 3 p d$ & 0.509 & 0.442 & 272.899 & $<0.001$ & 0.133 & 0.006 & $<0.001$ \\
\hline$l d h$ & 0.052 & 0.051 & 16.690 & 0.016 & 0.016 & 0.006 & 0.022 \\
\hline Ldh & 0 & 0 & - & - & - & - & - \\
\hline$M d h$ & 0.063 & 0.061 & 25.534 & 0.002 & 0.026 & 0.006 & 0.002 \\
\hline$M e-1$ & 0.119 & 0.108 & 188.268 & $<0.001$ & 0.093 & 0.006 & $<0.001$ \\
\hline$M e-2$ & 0 & 0 & - & - & - & - & - \\
\hline$M p i$ & 0.516 & 0.506 & 86.542 & $<0.001$ & 0.020 & 0.006 & 0.002 \\
\hline Pep-vl-1. & 0.448 & 0.409 & 238.027 & $<0.001$ & 0.089 & 0.006 & $<0.001$ \\
\hline Pep-vl-2 & 0 & 0 & - & - & - & - & - \\
\hline Pep-lgg & 0.630 & 0.502 & 502.686 & $<0.001$ & 0.203 & 0.006 & $<0.001$ \\
\hline Pep-pp & 0.714 & 0.694 & 102.146 & $<0.001$ & 0.028 & 0.006 & $<0.001$ \\
\hline $6 P g d$ & 0.265 & 0.259 & 40.213 & 0.001 & 0.021 & 0.006 & 0.001 \\
\hline Pgm-1 & 0.072 & 0.071 & 40.252 & $<0.001$ & 0.024 & 0.006 & $<0.001$ \\
\hline Pgm-2 & 0.355 & 0.284 & 486.091 & $<0.001$ & 0.200 & 0.006 & $<0.001$ \\
\hline Pgi & 0.520 & 0.410 & 590.180 & $<0.001$ & 0.212 & 0.006 & $<0.001$ \\
\hline$S d h$ & 0 & 0 & - & - & - & - & - \\
\hline$X d h$ & 0.668 & 0.635 & 234.446 & $<0.001$ & 0.049 & 0.006 & $<0.001$ \\
\hline
\end{tabular}

sampling error alone $(\mathrm{p}=0.024>\alpha=0.05 / 18=0.003)$. The remaining 17 loci all showed significant differentiation, with between about $1 \%$ (Aat-2, Mpi, $6 \mathrm{Pgdh}$ ) and $20 \%$ (Pep-lgg, Pgm-2, PgI) of the genetic variation arising from between-collection differences (these figures estimated from $G_{S T}-G_{S T \text {,null }}$ ).

Matrices of genetic distance between all pairwise collection comparisons were calculated by both Nei's (1978) and Rogers' (1972) measures. A UPGMA tree was derived from Nei's distances (Fig. 2) and a Wagner tree from Rogers' distances (using Sabella pavonina as an outgroup) (Fig. 3).

The UPGMA-derived dendrogram of Nei's (1978) genetic distances shows (1) that the Australian collections are, as expected from the earlier analyses, very closely related to one another, (2) that the Mediterranean collections are closely related to one another, and (3) that the Atlantic collection is more closely related to the Mediterranean collections than are the Australian collections.

The UPGMA tree separates the Australian collections from the European collections, because the Atlantic and Mediterranean collections are more closely related to one another than either is to the Australian collections. However, the Australian collections are more closely related to the Mediterranean collections (mean genetic distance $0.040, n=9$, range 0.029 to 0.050 ) than to the Atlantic collection (mean genetic distance $0.084, \mathrm{n}=3$, range 0.082 to 0.086 ). In fact, the Australian collections are more closely related to the Alicante collection (mean genetic distance $0.032, \mathrm{n}=3$, range 0.029 to 0.037 ) than to Carteau or Marseille (mean genetic distance 0.044, $\mathrm{n}=6$, range 0.038 to 0.050 )

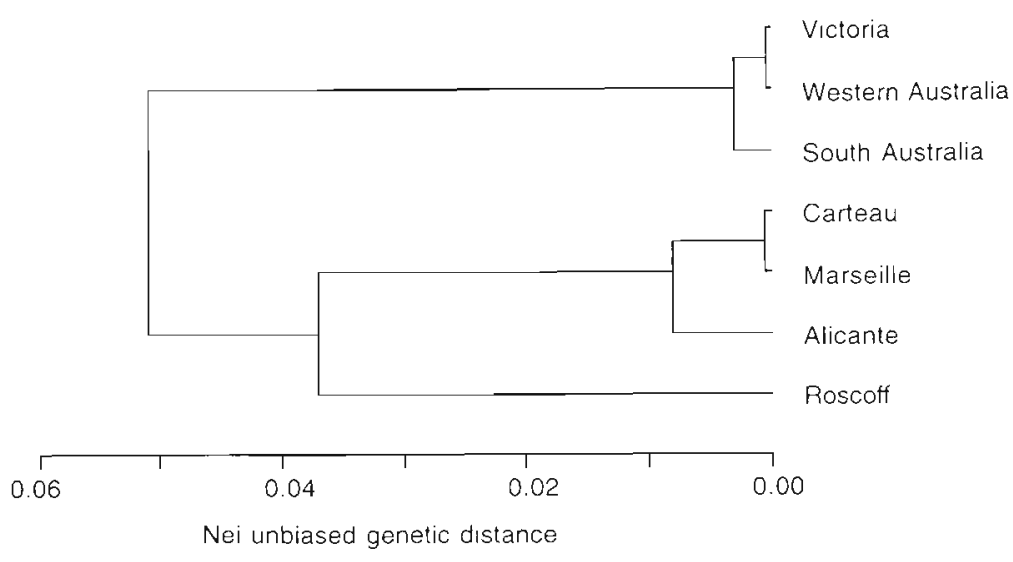

Fig. 2. Genetic relationships among Sabella spallanzani populations derived from a matrix of Nei's (1978) unbiased genetic distance between populations (using all 23 loci) and UPGMA clustering 
The Wagner tree of Rogers' (1972) distances with Sabella pavonina as an outgroup (Fig 3) shows that (1) the Australian collections are very closely related to one another and a little separate from other $S$. spallanzanii collections, (2) the Mediterranean collections are closely related to one another, and (3) the Australian collections are more closely related to the Alicante collection than to any other collection.

The main difference between the UPGMA tree and the Wagner tree concerns the placement of the Australian collections. In the UPGMA tree they cluster away from the European collections; in the Wagner tree they cluster with the Alicante collection. The close relationship between the Alicante and Australian populations has already been noted. A basic difference between UPGMA and the Wagner trees is that the former, unlike the latter, assumes a constant rate of evolution. It may well be that in the case of an introduced species, which has gone through a bottleneck leading to the loss of some genetic variation and therefore accelerated change in gene frequencies, the Wagner tree better represents population relationships by permitting an enhanced rate of evolution in the introduced populations.

The greater resemblance of the Australian collections to the Mediterranean collections than to the Atlantic collection can also be seen by looking at individual loci. The 3 most differentiated loci (Pep-Igg, $P g m-2$, and $P g i$, all with $G_{S T}$ values of around 0.20 , see Table 6) may be considered as examples.

With respect to Pep-lgg, the dominant European allele, Pep-lgg*90, has a frequency of about 0.82 in the Atlantic, 0.52 in the Mediterranean, but only 0.07 in the Australian collections. The common Australian allele, Pep$\lg g^{*} 100$, with a mean frequency of 0.56 , has a frequency of around 0.45 in the Mediterranean collections but only 0.19 in the Atlantic collection. With respect to Pgm-2, the Australian collections were monomorphic for Pgm$2 * 100$, which, while the most common allele in all European collections, was more abundant in the Mediterranean collections (frequency 0.68 ) than in the Atlantic collection (0.52). With respect to Pgi, the Australian collections were almost monomorphic for an allele $\left(P g i^{*} 100\right)$ that was the most common in the Mediterranean (frequency 0.53 ) but was only the second most common allele in the Atlantic collection (frequency 0.23).

\section{Sabella spallanzanii and S. pavonina: genetic relationships}

Sabella spallanzanii and S. pavonina are genetically very distinct. While only 10 specimens of S. pavonina

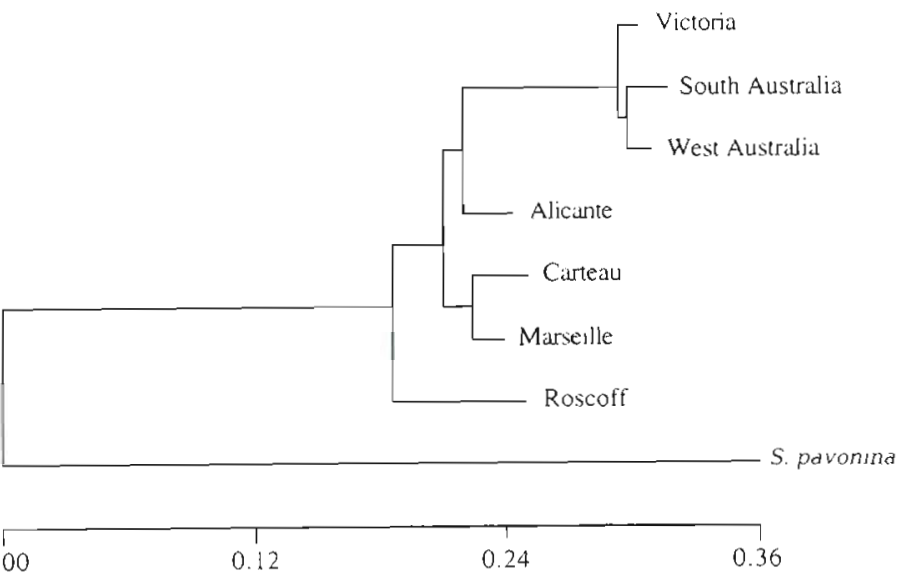

Rogers' (1972) genetic distance from root

Fig. 3. Genetic relationships among Sabella spallanzanii populations derived from a matrix of Rogers' (1972) genetic distanco bctwecn populations (using all 23 loci). The dendrogram is a Wagner tree rooted by the out-group $S$. pavonina

were examined (and more than 500 S. spallanzanii), fixed differences were observed at 9 loci (EstD-2, G3pdh, Me-1, Mpi, Pep-vl-1, Pep-vl-2, Pep-lgg, Sdh, $X d h$ ) and nearly fixed differences at 3 further loci (Aat1. Aat-2, Pgm-1). This genetic distinctiveness is not caused by allopatric separation of populations: the $S$. pavonina collection was sampled from Roscoff where it was sympatric with the sampled $S$. spallanzanii population.

The mean Nei (1978) genetic distance between Sabella spallanzanii and S. pavonina is 0.996 ( $n=7$. range 0.987 to 1.025 ), while the mean genetic distance among the different $S$ spallanzanii collections was only 0.035 ( $n=21$, range 0.000 to 0.086$)$. The mean genetic distance between Australian and European collections of $S$. spallanzanii was $0.051(n=12$, range 0.029 to 0.086 ). The separation of $S$. pavonina from the S. spallanzanii populations is also shown by the Wagner tree of Rogers' genetic distance (Fig. 3). The similarity of the European and Australian collections of $S$. spallanzanil to one another, plus the distinctiveness of S. pavonina, indicates that the Australian collections are indeed $S$. spallanzanii and not another Sabella species

\section{DISCUSSION}

The genetic data indicate that the Australian specimens are indeed Sabella spallanzanii, and that they derive from 1 or more European introductions. The species has been described from the Ionian Sea as a protandric sequential hermaphrodite /Giangrande \& 
Petraroli 1994), and the good fits to Hardy-Weinberg equilibrium found here suggest that selfing is rare, if indeed it occurs at all.

\section{Comparing levels of variation among populations}

Sabella spallanzanii is clearly very variable genetically. The average heterozygosity per locus, over the 23 examined loci, for the Mediterranean collections was about 0.25 . This is about twice as high as the average for 370 species of marine and terrestrial invertebrates recorded by Ward et al. (1992) as 0.122 , although perhaps $10 \%$ of invertebrates are even more variable than $S$. spallanzanii.

Among the European collections, the Atlantic Roscoff collection was less variable than the Mediterranean collections in terms of mean heterozygosity, average numbers of alleles per locus, and percentage of polymorphic loci. A total of 22 alleles were found in the Mediterranean collections but not in the Roscoff collection, however no alleles were found in the Roscoff that were not found in the Mediterranean. This asymmetric pattern cannot simply be attributed to more Mediterranean animals having been sampled, since even the individual Mediterranean collections, with sample sizes smaller than the Roscoff collection, showed Mediterranean-specific alleles (Carteau, 17 Marseille, 17; Alicante, 20).

While these alleles were all quite rare-generally having frequencies less than 0.10 and therefore contributing relatively little to heterozygosity - their presence in the Mediterranean and absence from the Atlantic suggests either that the species was originally native to the Mediterranean and lost rare alleles in the process of spreading to the Atlantic seaboard, or that Mediterranean populations have maintained higher population sizes and therefore more variation than the Atlantic populations. The latter was suggested by Bembo et al. (1996) to account for their similar finding of greater allozyme heterozygosity in Mediterranean anchovies (Engraulis encrasicolus) than in an Atlantic sample. When higher mitochondrial DNA diversity was found in Mediterranean populations of Mytilus galloprovincialis than in Atlantic populations, Quesada et al. (1995a) suggested that Pleistocene refugia in the Mediterranean maintained higher population sizes than Atlantic populations during glacial maxima. However, despite some allozyme gene-frequency differences (Quesada et al. 1995b), there were no differences in overall allozyme heterozygosity between the Mediterranean and Atlantic populations of $M$. galloprovincialis $(\mathrm{H}$. Quesada pers. comm.) In any case. these apparent differences in variability between Atlantic and Mediterranean populations of Sabella spallanzanii need to be confirmed with additional sampling, especially from the Atlantic.

The Australian collections were somewhat less variable than the European collections. While the mean numbers of alleles per locus were similar to those of the Roscoff collection, at around 2.0 (compared with about 2.7 for the Mediterranean), sample sizes were substantially higher, which would have increased the probability of rare alleles being detected. Number of alleles and percentage polymorphism are more sample-size dependent than average heterozygosity per locus, and it is this latter parameter that is the best single parameter for estimating levels of genetic variation. The Australian collections had an average heterozygosity about $18 \%$ less than the average of the 4 European collections, and about $20 \%$ less than that of the 3 Mediterranean collections. Two loci in particular $-P g m-2$ and $P g i-$ show striking losses in variation. Pgm-2 is monomorphic in Australian collections but highly polymorphic in all European collections examined. Pgi shows little variation in Australian collections but is highly variable in European collections. It is clear that the introduction from Europe into Australia was accompanied by the loss of some genetic variation.

Sabella spallanzanii produces large numbers of planktonic larvae and so can increase in population size very rapidly after an introduction to a favourable environment. Nei et al. (1975) and Chakraborty \& Nei (1977) showed that the reduction in heterozygosity depends not only on the size of a population bottleneck but also on the rate of recovery. Hence, bottleneck effects in species with high fecundity and potentially high rates of population increase, such as S. spallanzanii, may be less marked than in species with low fecundity. Some introductions of species with planktonic larvae have been accompanied by little or no detectable loss in genetic variation (e.g. the zebra mussel Dreissena polymorpha to North America, Boileau \& Hebert 1993, Marsden et al. 1995), while others have shown large losses (e.g. the northern Pacific seastar Asterias amurensis to Tasmania lost about $35 \%$ of its genetic variation, Ward \& Andrew 1995). Differences in the amount of variation lost in species with similar life histories may reflect differences in the original founding population sizes or perhaps number of introductions. The loss of 18 to $20 \%$ of variation in introduced populations of $S$ spallanzanii could be attributable both to a founder effect and to small numbers of animals in the first few generations.

The transport mechanism that brought Sabella spallanzanii to Australia from Europe is unclear Certainly the species has been observed attached to ships' hulls (Clapin \& Evans 1995, S. Rainer pers. comm.), and they probably can remain attached to at least slow-moving vessels. Specimens have survived short periods of high 
temperature $\left(12 \mathrm{~h}\right.$ at $\left.30^{\circ} \mathrm{C}\right)$, which would increase their chances of surviving transport through tropical waters, although stress observed at $27^{\circ} \mathrm{C}$ makes it unlikely that they will ever colonise warm tropical waters (Clapin 1996). It is also possible that they can be transported as larvae in ballast water, but their lecithotropic nature (Giangrande \& Petraroli 1994, Rouse \& Fitzhugh 1994) is likely to mean that their larval stages are relatively short-lived and thus unable to survive long-distance shipping. Thus transport from Europe to Australia is more likely to have been as hull-attached juvenile or adult worms than as larvae.

\section{Genetic relationships among populations}

There are at least 4 major infestations of Sabella spallanzanii in Australia (Cockburn Sound and Albany, Western Ausiraliad Adelaide, South Australiá; Port Phillip Bay, Victoria). Do these represent separate introductions from 4 different sources? The answer to this question seems to be no, as the 3 studied Australian populations were genetically very similar to one another, with no statistically significant differences in gene frequency. This implies a single source, because otherwise the significant heterogeneity observed among the European populations studied would be reflected in heterogeneity among Australian populations.

The indications are that the source was somewhere in the Mediterranean Sea. This is suggested both by the mean Nei (1978) genetic distance between the Mediterranean and Australian collections being only 0.040 (compared with 0.084 for the Atlantic and Australian collections) and by inspection of allele frequencies at individual loci. Of course, this conclusion bears the caveat that only 1 Atlantic population, Roscoff, was examined, and it assumes that other Atlantic populations would be more like Roscoff than like the Mediterranean populations. Importation from the Alicante region is more likely than from the Marseille region, although there are of course a large number of as-yetunstudied putative source ports.

The next question is whether there was a single or multiple introduction from the European source. It could be argued that if there had been independent introductions to different parts of Australia, the likely small size of the founding populations (hull-borne juveniles or adults) would be expected to lead to spatial genetic heterogeneity unless there was subsequent widespread gene flow. An alternative scenario, a single introduction followed by a spread to other sites, could account for the relative genetic uniformity of the Australian populations, However, this scenario posits gene flow to the secondary sites, and if there were such gene flow, then this could lead to homogenisation of allele frequencies among sites resulting from separate introductions. The present data therefore do not allow clear discrimination between the single introduction and multiple introduction hypotheses. Note that within-Australia gene flow could be mediated not only by individuals attached to the hulls of coastal shipping but also by ballast-water transport of the short-lived eggs and larvae, as shipping times among Australian ports are much less than between European and Australian ports. The extent of gene flow among Australian ports would be expected to be greater than from Europe to Australia.

If there was a single invasion of southern Australian waters, followed by a spread, then which area received that first invasion? This cannot be answered from the present genetic data, but Clapin \& Evans (1995) suggest, from examining museum material, that Sabella spallanzanii may have been in the Albany region of Western Australia as early as 1965. This is currently the oldest record in Australia, and it is possible that, while Albany is only a minor port berthing small and medium-sized vessels, it was the first port of introduction.

In principal, if there were a single introduction (say to Albany), followed by secondary colonisations from that original introduction, then the population resulting from the first colonisation should show more genetic variation than the second. If there were a linear sequence of colonisations, with the third introduction deriving from the second, and so on, then each subsequent introduction would be expected to result in the loss of further variability. The allozyme approach we used is not powerful enough to examine such expectations, since the numbers of alleles per locus, averaging 2 to 2.5 , were too low. However, the analysis of variation at microsatellite loci could well lead to very interesting results, since for such loci the numbers of alleles can be as high as 40 or 50 . With so many alleles, the consecutive loss of alleles with subsequent introductions should be measurable. The examination of 5 to 10 such loci per species could well revolutionise our understanding of the routes taken by accidental introductions of both marine and terrestrial organisms.

Acknowledgements. This work was funded by the CSIRO Centre for Research into Introduced Marine Pests (CRIMP). We wish to thank all those who helped with collectung the samples: Kirrily Moore, Marnie Nelson, Sebastian Rainer, Charles Sutherland (CSIRO Marine Research, Hobart, Tasmania), Dave Evans (CSIRO Marine Research, Marmion, Western Australia), Patrick Hone (SARDI Aquatic Sciences Centre, West Beach, South Australia), Helmut Zibrowius (Station Mārine d'Endoume, Marseille), Franck Gentil (Station Biologique, Universıté P. \& M. Curie, Roscoff), and Pablo Sanchez (Universidad de Alicante, Alicante). We also thank Gary Poore (Victorian Museum) for transporting the Marseille and Carteau samples to Australia, Helmut Zibrowius and Sebastian Rainer for their interest and comments on this 
work, and Nick Elliott, Chad Hewitt, Kerstin Johannesson, Vivienne Mawson, Peter Rothlisberg and an anonymous referee for their comments on the manuscript.

\section{LITERATURE CITED}

Bembo DG, Carvalho GR, Cingolani N, Pitcher TJ (1996) Electrophoretic analysis of stock structure in northern Mediterranean anchovies, Engraulis encrasicolus. ICES J Mar Sci 53:115-128

Bitar G (1987) Étude des peuplements benthiques littoraux des côtes atlantiques et mediterranéennes du Maroc Impact de la pollution, comparaisons biogéographiques. These, Univ. d'Aix-Marseille 2

Boileau MG. Hebert PDN (1993) Genetics of the zebra mussel (Dreissena polymorpha) in populations from the Great Lakes region and Europe. In: Nalepa TF, Schloesser D (eds) Zebra mussels: biology, impacts and control. Lewis Publishers, Ann Arbor, MI

Carey JM. Watson JE (1992) Benthos of the muddy bottom habitat of the Geelong Arm of Port Phillip Bay, Victoria, Australia. Vic Nat 109(6):196-202

Chakraborty R, Nei M (1977) Bottleneck effects on average heterozygosity and genetic distance with the stepwise mutation model. Evolution 31:347-356

Clapin G (1996) The filtration rate, oxygen consumption and biomass of the introduced polychaete Sabella spallanzanii Gmelin within Cockburn Sound. BSc Hons thesis, Edith Cowan University, Perth, Western Australia

Clapin G, Evans DR (1995) The status of the introduced marine fanworm Sabella spallanzanii in Western Australia: a preliminary investigation. Centre for Research on Introduced Marine Pests. Tech Rep 2, CSIRO Division of Fisheries, Hobart

Ewer DW (1946) Sabella pavonna Savigny Var. bicoronata Hornell and the genus Spirographis Viviani (Polychaeta, Sabellidae). J Mar Biol Assoc UK 26:426-431

Giangrande A, Petraroli A (1994) Observations on reproduction and growth of Sabella spallanzanii (Polychaeta Sabellidae) in the Mediterranean Sea. In: Dauvin JC, Laubier L, Reish DJ (eds) Actes dé la 4ème Conférence internationale des Polychaètes. Mem Mus Natl Hist Nat, Paris 162:51-56

Hebert PDN, Beaton MJ (1989) Methodologies for allozyme analysis using cellulose acetate electrophoresis. Helena Laboratories, Beaumont, TX

This article was presented by T. Fenchel (Senior Editorial Advisor), Helsinger, Denmark
Hochberg Y (1988) A sharper Bonferroni procedure for multiple tests of significance. Biometrika 75:800-802

Koechlin N (1977) Installation d'une épifaune a Spirographis spallanzanii Viviani, Sycon ciliatum Fabricius et Ciona intestinalis (L.) dans le port de plaisance de Lézardrieux (Côtes du Nord). Cah Biol Mar 18:325-337

Marsden JE, Spidle A, May B (1995) Genetic similarity among zebra mussel populations within North America and Europe. Can J Fish Aquat Sci 52:836-847

Nei M (1973) Analysis of gene diversity in subdivided populations. Proc Natl Acad Sci USA 70:3321-3323

Nei M (1978) Estimation of average heterozygosity and genetic distance from a small number of individuals Genetics 89:583-590

Nei M. Maruyama T, Chakraborty R (1975) The bottleneck effect and genetic variability in populations. Evolution 29 $1-10$

Perkins TH, Knight-Jones $P$ (1991) Towards a revision of the genera Sabella and Bispira (Sabellidae). Ophelia (Suppl) 5:698

Quesada H, Wenne R, Skibinski DOF (1995a) Differential introgression of mitochondrial DNA across species boundaries within the marine mussel genus Mytilus. Proc R Soc Lond Ser B Biol Sci: 262:51-56

Quesada H, Zapata C, Alvarez G (1995b) A multilocus allozyme discontinuity in the mussel Mytilus galloprovincialis: the interaction of ecological and life-history factors. Mar Ecol Prog Ser 116:99-115

Richardson BJ, Baverstock PR, Adams M (1986) Allozyme electrophoresis: a handbook for animal systematics and population studies. Academic Press, New York

Roff DA, Bentzen P (1989) The statistical analysis of mitochondrial DNA polymorphisms: $\chi^{2}$ and the problem of small samples. Mol Biol Evol 6:539-545

Rogers JS (1972) Measures of genetic similarity and genetic distance. Stud Genet 7:145-153

Rouse GW, Fitzhugh K (1994) Broadcasting fables: is external fertilization really primitive? Sex, size, and larvae in sabellid polychaetes. Zool Scr 23:271-312

Ward RD, Andrew J (1995) Population genetics of the northern Pacific seastar Asterias amurensis Lutken (Echinodermata: Asteriidae): allozyme differentiation among Japanese, Russian, and recently introduced Tasmanian populations. Mar Biol 124:99-109

Ward RD, Skibinski DOF, Woodwark M (1992) Protein heterozygosity, protein structure, and taxonomic differentiation. Evol Biol 26:73-159

Manuscript first received: January 22, 1997

Revised Version accepted: April 4, 1997 